\title{
Cost-effectiveness of steroid (methylprednisolone) injections versus anaesthetic alone for the treatment of Morton's neuroma: economic evaluation alongside a randomised controlled trial (MortISE trial)
}

Rhiannon Tudor Edwards ${ }^{1 *}$, Seow Tien Yeo ${ }^{1}$, Daphne Russell², Colin E Thomson ${ }^{3}$, lan Beggs ${ }^{4}$, J N Alastair Gibson ${ }^{5}$, Diane McMillan ${ }^{3}$, Denis J Martin ${ }^{6}$ and lan T Russell ${ }^{2}$

\begin{abstract}
Background: Morton's neuroma is a common foot condition affecting health-related quality of life. Though its management frequently includes steroid injections, evidence of cost-effectiveness is sparse. So, we aimed to evaluate whether steroid injection is cost-effective in treating Morton's neuroma compared with anaesthetic injection alone.

Methods: We undertook incremental cost-effectiveness and cost-utility analyses from the perspective of the National Health Service, alongside a patient-blinded pragmatic randomised trial in hospital-based orthopaedic outpatient clinics in Edinburgh, UK. Of the original randomised sample of 131 participants with Morton's neuroma (including 67 controls), economic analysis focused on 109 (including 55 controls). Both groups received injections guided by ultrasound. We estimated the incremental cost per point improvement in the area under the curve of the Foot Health Thermometer (FHT-AUC) until three months after injection. We also conducted cost-utility analyses using European Quality of life-5 Dimensions-3 Levels (EQ-5D-3L), enhanced by the Foot Health Thermometer (FHT), to estimate utility and thus quality-adjusted life years (QALYS).

Results: The unit cost of an ultrasound-guided steroid injection was $£ 149$. Over the three months of follow-up, the mean cost of National Health Service resources was $£ 280$ for intervention participants and $£ 202$ for control participants - a difference of $£ 79$ [bootstrapped 95\% confidence interval (Cl): $£ 18$ to $£ 152$ ]. The corresponding estimated incremental cost-effectiveness ratio was $£ 32$ per point improvement in the FHT-AUC (bootstrapped 95\% Cl: $£ 7$ to $£ 100$ ). If decision makers value improvement of one point at $£ 100$ (the upper limit of this $\mathrm{Cl}$ ), there is $97.5 \%$ probability that steroid injection is cost-effective. As EQ-5D-3L seems unresponsive to changes in foot health, we based secondary cost-utility analysis on the FHT-enhanced EQ-5D. This estimated the corresponding incremental cost-effectiveness ratio as $£ 6,400$ per QALY. Over the recommended UK threshold, ranging from $£ 20,000$ to $£ 30,000$ per QALY, there is $80 \%-85 \%$ probability that steroid injection is cost-effective.

(Continued on next page)
\end{abstract}

\footnotetext{
*Correspondence: r.t.edwards@bangor.ac.uk

'Bangor University, Centre for Health Economics and Medicines Evaluation (CHEME), School of Healthcare Sciences, College of Health and Behavioural Sciences (CoHaBS), Ardudwy Hall, Normal Site, Bangor LL57 2PZ, Gwynedd, UK Full list of author information is available at the end of the article
} 
(Continued from previous page)

Conclusions: Steroid injections are effective and cost-effective in relieving foot pain measured by the FHT for three months. However, cost-utility analysis was initially inconclusive because the EQ-5D-3L is less responsive than the FHT to changes in foot health. By using the FHT to enhance the EQ-5D, we inferred that injections yield good value in cost per QALY.

Trial registration: Current Controlled Trials ISRCTN13668166

Keywords: Morton's neuroma, Interdigital plantar nerves, Methylprednisolone, Steroid injection, Foot health, Cost-effectiveness analysis, Cost-utility analysis, Quality-adjusted life years

\section{Background}

Morton's neuroma is the common descriptive term for a benign neural swelling of one of the interdigital plantar nerves. The condition most commonly affects the nerves in the second and third interspaces and is more common in middle-aged women [1,2]. Causal factors may include high heeled or ill-fitting shoes, high impact athletic activities such as jogging and direct trauma [3,4]. Symptoms from Morton's neuroma may include paraesthesia, burning, numbness and pain $[1,2,4]$. The clinical and economic significance of Morton's neuroma is that prolonged disabling foot pain can lead to limitations in daily activities and sickness absence [2]. Treatment options include insertion of insoles into footwear, steroid injections to manage foot pain, and eventual surgery [5-7].

Steroid injections are common second-line interventions but evidence of effectiveness and cost-effectiveness is sparse [2]. This paper presents the economic findings of a randomised trial of steroid injection in the treatment of Morton's neuroma [8]. This trial found that in comparison with the control group, foot health in the corticosteroid group was significantly better at one and three months: at three months the mean difference was 14.1 points on the Foot Health Thermometer (95\% confidence interval: 5.5 to 22.8 points; $\mathrm{p}=0.002$ ). Corticosteroid injections also improved pain, function and general health one and three months after injection. The size of the neuroma on ultrasound did not significantly influence the effect of treatment.

Since then there has been little change in clinical practice; steroid injections remain a second-line treatment for Morton's neuroma. The current systematic review for the Cochrane Library [9] stresses the lack of clinical evidence for this common condition. Hence, our primary economic aim was to investigate the cost-effectiveness of steroid injection compared with anaesthetic injection alone in the treatment of Morton's neuroma in reducing foot pain over three months.

\section{Methods}

\section{Study design and interventions}

The economic evaluation took place alongside the trial known as 'Morton's neuroma: Injected Steroids Effective?'
(MortISE) [Registration: http://www.controlled-trials.com/ ISRCTN13668166], a patient-blinded pragmatic randomised trial designed to compare the effectiveness of steroid injections with anaesthetic injections in alleviating pain and other effects of Morton's neuroma [8]. The trial included 131 patients with clinical symptoms of Morton's neuroma; their mean age was 53 years, and 111 were female. We randomised participants between an intervention group receiving corticosteroid and anaesthetic injections [ $1 \mathrm{ml}$ methylprednisolone $(40 \mathrm{mg}$ ) and $1 \mathrm{ml} 2 \%$ lignocaine] and a control group receiving anaesthetic alone (2 $\mathrm{ml} 1 \%$ lignocaine). We kept trial participants blind to the type of injection they received. Though we had intended also to keep the radiologist blind, this proved impractical. Nevertheless the radiologist played no other part in the study [8].

Participants completed measures at baseline before treatment and at follow-up clinics one and three months after randomisation. We chose the interval of one month to maximise clinical benefit and identify any adverse events following injection; and that of three months to estimate medium-term benefit. We collected the following outcome data for the trial: neuroma size, foot pain and disability by the Foot Health Thermometer (FHT) and the Manchester Foot Pain and Disability Schedule, pain by the Multidimensional Affect and Pain Survey and health-related quality of life by the European Quality of life-5 Dimensions-3 Levels (EQ-5D-3L). The primary outcome was participants' assessment of their foot health by the FHT at three months after injection.

The Lothian Research Ethics Committee approved the study. All participants provided written informed consent before starting the study.

\section{Economic evaluation}

We conducted an economic evaluation three months after randomisation of participants. Our aim was to evaluate steroid injections for the treatment of Morton's neuroma. To be comprehensive we addressed both costeffectiveness, which focuses on foot health, through the FHT, and cost-utility, which addresses health-related quality of life. For the latter, the National Institute for Health and Care Excellence (NICE) in the UK has recommended 
the use of quality-adjusted life years (QALYs) as the measure of health benefit for economic analysis as it allows comparisons across different clinical conditions, unlike condition-specific outcomes like the FHT [10].

As it is not easy to impute missing cost data, we based economic analysis of this trial on a smaller sample than the effectiveness analysis. Data for the costs of service use were incomplete for 10 participants in the intervention group and 12 participants in the control group, though we had included 13 of them (5 intervention and 8 control) in effectiveness analyses. We had full economic data and enough baseline and follow-up data to impute QALYs and the area under the curve of the Foot Health Thermometer (FHT-AUC) for 109 participants 54 in the intervention group and 55 participants in the control group. This represented $89 \%$ of the 122 participants in the main effectiveness analysis [8].

\section{Measurement of costs}

We examined costs from the perspective of the National Health Service $[11,12]$. We estimated direct primary and secondary care use from hospital records and participants' self-reported client service receipt inventories [13] at one and three months. Research resource constraints prevented us from asking participants to complete client service receipt inventories at baseline. At one and three months the client service receipt inventories asked patients to recall all contacts with primary care, attendances at emergency departments, and inpatient stays. From hospital records we gathered data on steroid and anaesthetic injections, outpatient visits, surgery, radiological imaging, and laboratory tests and investigations. We derived unit costs of these service contacts in pounds Sterling from national sources $[14,15]$ (Table 1$)$.

We inflated these costs extracted from Department of Health [14] and Curtis and Netten [15] from 2004-5 to 2011-2 using Hospital \& Community Health Service inflation indices [16]. We obtained costs of steroid and anaesthetic drugs from the British National Formulary version 52 [17] and inflated them to 2011-2 prices. During the three-month follow-up period, three participants (two in the intervention group and one in the control group) underwent surgical procedures for gastroenterological and gynaecological conditions. As these inpatient costs did not relate to foot health, we did not include them in our analysis.

\section{Measurement of effectiveness}

Our primary analysis was a cost-effectiveness analysis $[10,18]$ using FHT-AUC, the area under the curve of FHT scores, to measure the outcome of the trial. The FHT is a validated visual analogue scale similar to the EQ-5D-3L thermometer with 0 representing worst possible foot health and 100 best possible foot health [19]. We corrected all effects for differences in baseline, thus improving the accuracy of estimated effects.

We undertook a cost-utility analysis using QALYs as the measure of effect. We estimated participant utilities by administering the EQ-5D-3L instrument [20] at baseline, one month and three months; combined them using the area under the curve method to calculate QALY gains over the three month study period; and

Table 1 Unit cost (£) and source of health service use in the UK ${ }^{a, b, c}$

\begin{tabular}{|c|c|c|c|}
\hline Health-care resource & Unit & Unit cost $(£)^{a}$ & Details and source \\
\hline Primary care contacts, e.g. general practitioner, practice nurse. & Consultation & 12.30 to 84.86 & Costed by profession $^{d}$ \\
\hline PAMs, e.g. physiotherapist, chiropodist, consultant radiologist & Consultation & 12.30 to 51.65 & Costed by profession $^{d}$ \\
\hline Hospital outpatient clinic e.g. orthopaedic, opthalmologist & Consultation & 103.31 to 110.69 & Costed by specialty ${ }^{\mathrm{d}, \mathrm{e}}$ \\
\hline Hospital outpatient consultation with ultrasound scan & Consultation with scan & 145.74 & $\begin{array}{l}\text { Cost including costs of consultant } \\
\text { radiologist lasting 30mins, nurse lasting } \\
30 \text { minutes of client contact and an } \\
\text { ultrasound scan }^{d, e}\end{array}$ \\
\hline Hospital outpatient consultation with no ultrasound scan & Consultation with no scan & 63.34 & Cost of consultation lasting $30 \mathrm{mins}^{\mathrm{e}}$ \\
\hline Inpatient hospital stay & Procedure & 154.96 & Costed by procedure ${ }^{e}$ \\
\hline Accident and emergency & Consultation & 28.29 & Costed by consultation $^{\mathrm{e}}$ \\
\hline $\begin{array}{l}\text { Steroid injection ( } 1 \mathrm{ml} \text { methylprednisolone }(40 \mathrm{mg} \text { ) and } 1 \mathrm{ml} \\
2 \% \text { Lignocaine) }\end{array}$ & Item & 3.74 & Costed by BNF entry ${ }^{f}$ \\
\hline Anaesthetic injection ( $2 \mathrm{ml}$ of $1 \%$ Lignocaine) & Item & 0.25 & Costed by BNF entry ${ }^{f}$ \\
\hline
\end{tabular}

Legend: Table 1 Unit cost of health service use in UK pounds sterling $(£)^{a}$, with source ${ }^{b, c}$.

PAMs: Professionals Allied to Medicines; BNF: British National Formulary.

${ }^{a}$ Cost year 2011/12.

${ }^{\mathrm{b}}$ National Health Service costs including salary, employers' costs, overheads and capital costs.

${ }^{c}$ Costs extracted from Curtis and Netten [11] and Department of Health [10] have been inflated from 2004/05 to 2011/12 using Hospital \& Community Health

Service inflation indices from Curtis [12].

${ }^{\mathrm{d}}$ From Curtis and Netten [11].

eFrom Department of Health [10].

fFrom BNF [13]. 
corrected for baseline EQ-5D-3L. We estimated the cost per QALY gain by dividing differences in cost by difference in QALYs and compared these with the thresholds recommended by NICE in the UK [10]. We did not discount costs or effects as the follow-up period was less than one year.

To quantify the uncertainty around the estimated incremental cost-effectiveness ratios, for both cost-effectiveness and cost-utility analyses, we ran a simulation of 5000 nonparametric bootstrapped iterations using MS Excel 2007. We used these to estimate confidence intervals (CIs) for incremental costs and incremental cost-effectiveness ratios and to construct a cost-effectiveness plane - a scatter plot of the joint distribution of incremental costs and effects - and a cost-effectiveness acceptability curve [21]. The cost-effectiveness acceptability curve displays the probability that an intervention is more cost-effective than the alternative across a range of thresholds of willingness to pay for a QALY [22,23].

\section{Sensitivity analyses}

To assess how dependent on our original assumptions our findings are, we undertook two forms of sensitivity analysis. Firstly, we recognised that the MortISE trial had adopted as its primary measure of benefit - the FHT, a Patient-Reported Outcome Measure with evidence of responsiveness to change [8]. In contrast, for our cost-utility analysis alongside MortISE we chose as primary measure of benefit - the EQ-5D-3L, a PROM with a strong theoretical basis for its economic validity $[24,25]$. However, as there are concerns that the use of three-point scales by EQ-5D-3L reduces its responsiveness to change [26,27], we regressed the EQ-5D-3L data from the MortISE participants as the dependent variable on their FHT data as the independent variable, with allocated treatment as the covariate. The resulting regression equation has two complementary functions: it converts participants' responses to the FHT into utilities on the original EQ-5D-3L scale, and it uses the greater discrimination achieved by the FHT to fill gaps in the simplistic three-point scales that characterised the original EQ-5D-3L. Though the custodians of the EQ-5D$3 \mathrm{~L}$ have recently sought to improve responsiveness by expanding scales to five points in the EQ-5D-5L [28], the fact that we conceived MortISE before then stimulated us to find another method of strengthening the EQ-5D-3L, which we call the FHT-enhanced EQ-5D.

Secondly, we recognised that the costing of steroid injections depended on the design of MortISE, which performed ultrasound scans on all trial participants; in the intervention group to guide the steroid injections, and in the control group to assess the neuroma and need for surgery. Though this design aimed to deliver best practice to the control group, it also had the explanatory aim
[29] of equalising the 'placebo effect' between groups; because both groups received ultrasound scans, we could blind them to whether their injection contained steroid or anaesthetic. For our second sensitivity analysis we made the more pragmatic assumption [29] that control participants could attend the outpatient clinic for review and injection without receiving an ultrasound scan. If so, the unit cost of their hospital appointment, previously $£ 145.74$ including ultrasound scan, would fall to $£ 63.34$ without ultrasound scan. We then added the unit cost of anaesthetic injection ( $£ 0.25)$ to yield a total cost of $£ 63.59$ for hospital appointment with anaesthetic injection but no ultrasound scan.

\section{Computing software}

We analysed data in SPSS version 16.0 (SPSS Inc., Chicago II, USA) and MS Excel 2007 (Microsoft Corporation, Redmond, Washington DC, USA).

\section{Results}

Baseline demographic characteristics of study participants Table 2 summarises the demographic characteristics of participants in the intervention and control groups at baseline. As in the main effectiveness paper [8], demographic variables were similar across intervention and control groups.

\section{Effectiveness}

The effectiveness results for our economic sample of 109 participants were similar to those for the slightly larger sample in the main effectiveness paper [8]: steroid injections significantly improved foot pain (by more than ten points on the FHT) at both one month $(\mathrm{p}=0.006)$ and three months $(\mathrm{p}=0.013)$; but had no significant effect on health-related quality of life as measured by EQ-5D-3L. Combining the results for each of the study time points (baseline, one month and three months) into areas under the curves and correcting for baseline, the intervention group had significantly better FHT-AUC than the control group, with a mean difference of 2.472 points (95\% CI: 0.986 to $3.958 ; \mathrm{p}=0.001$ ). However, the mean QALY gain did not differ significantly between groups; the estimated improvement in QALY from using steroid injections was only 0.0038 ( $95 \% \mathrm{CI}$ : -0.0146 to $0.0221, \mathrm{p}=0.68$ ) [Table 3], which is 1.4 quality-adjusted life days.

\section{Frequency and cost of steroid and anaesthetic injections}

We estimated the costs of an outpatient visit for ultrasound-guided steroid injection as £149.48. This comprised $£ 3.74$ for the steroid injection and $£ 145.74$ for the outpatient appointment, including 30 minutes of consultant and nurse time, and an ultrasound scan. All intervention participants received one ultrasound-guided steroid injection at baseline. 
Table 2 Baseline demographic characteristics of the participating patients with Morton's neuroma ${ }^{a}$

\begin{tabular}{|c|c|c|}
\hline & $\begin{array}{l}\text { Control group } \\
(n=55)\end{array}$ & $\begin{array}{l}\text { Steroid group } \\
(n=54)\end{array}$ \\
\hline \multicolumn{3}{|l|}{ Gender } \\
\hline Male & $9(16 \%)$ & 10 (19\%) \\
\hline Female & $46(84 \%)$ & 44 (81\%) \\
\hline \multicolumn{3}{|l|}{ Age (year) } \\
\hline Mean (SD); range & $52.6(12.3) ; 26-76$ & $54.3(12.2) ; 28-79$ \\
\hline \multicolumn{3}{|l|}{ Current smoker } \\
\hline Smoker & $6(11 \%)$ & $6(11 \%)$ \\
\hline Ex-smoker & $17(31 \%)$ & 17 (32\%) \\
\hline Non-smoker & $32(58 \%)$ & $30(56 \%)$ \\
\hline Missing & $0(0 \%)$ & $1(2 \%)$ \\
\hline \multicolumn{3}{|l|}{ Employment } \\
\hline In employment & $34(62 \%)$ & $33(61 \%)$ \\
\hline Retired & $12(22 \%)$ & $12(22 \%)$ \\
\hline Housework & $7(13 \%)$ & $5(9 \%)$ \\
\hline Other & $2(4 \%)$ & $3(6 \%)$ \\
\hline Missing & $0(0 \%)$ & $1(2 \%)$ \\
\hline \multicolumn{3}{|l|}{ Education } \\
\hline \multicolumn{3}{|c|}{$\begin{array}{l}\text { Continued after the } \\
\text { minimum school } \\
\text { leaving age }{ }^{b}\end{array}$} \\
\hline Yes & $31(56 \%)$ & $32(60 \%)$ \\
\hline No & $24(44 \%)$ & 21 (39\%) \\
\hline Missing & $0(0 \%)$ & $1(2 \%)$ \\
\hline \multicolumn{3}{|c|}{ Degree or equivalent ${ }^{\mathrm{b}}$} \\
\hline Yes & $18(33 \%)$ & 19 (35\%) \\
\hline No & $37(67 \%)$ & 35 (65\%) \\
\hline Missing & $0(0 \%)$ & $0(0 \%)$ \\
\hline \multicolumn{3}{|l|}{$\begin{array}{l}\text { Body Mass Index } \\
\text { (BMI) }\left(\mathrm{kg} / \mathrm{m}^{2}\right)\end{array}$} \\
\hline Mean (SD); range & 27.7 (4.2); 21.3-40.7 & $27.7(5.4) ; 21.1-52.1$ \\
\hline
\end{tabular}

${ }^{a}$ Figures are numbers (percentages) of the patients unless stated otherwise. Percentages may not add to 100 due to rounding.

${ }^{\mathrm{b}}$ These two categories are not mutually exclusive.

We estimated the costs of an outpatient visit for an ultrasound-guided anaesthetic injection as $£ 145.99$. This comprised $£ 0.25$ for the anaesthetic injection and the same $£ 145.74$ for the outpatient appointment. All 55 control participants received one anaesthetic injection at baseline but two also received a steroid injection at follow up for the pain they were still experiencing (Table 4).

\section{Frequency and cost of service use by participants}

Tables 4 and 5 show the frequency and cost of contacts with National Health Service primary and secondary care by participants in intervention and control groups. Table 5 shows mean costs of all services received by intervention and control participants over the three month follow-up period. These included ultrasoundguided injections, outpatient visits and primary care consultations.

Table 5 shows mean cost differences and bootstrapped 95\% CIs. The mean total cost per participant was $£ 280.37$ for the intervention group and $£ 201.69$ for the control group. The difference in mean cost between the intervention and control groups was $£ 78.67$ (bootstrapped 95\% CI: $£ 18.25$ to $£ 152.34$ ).

\section{Primary cost-effectiveness analysis}

The incremental cost-effectiveness ratio was $£ 31.83$ per point improvement in FHT-AUC (bootstrapped 95\% CI from $£ 6.79$ to $£ 99.94$ ) - the result of dividing the difference in mean cost between intervention and control groups $(£ 280.37-£ 201.69=£ 78.68$; Table 5$)$ by the difference in mean FHT-AUC between the two groups $(14.748-12.276=2.472$; Table 3$)$.

\section{Incorporating uncertainty}

To assess uncertainty around incremental cost-effectiveness ratio estimates, we ran 5,000 bootstrap replications; Figure 1A shows the corresponding cost-effectiveness plane. Most points (99.54\%) fall within the North East quadrant, where the intervention is both more costly and more effective than the control group; 19 bootstrapped replications $(0.38 \%)$ fall in the South East quadrant (less costly, more effective) and the remaining four bootstrapped replications $(0.08 \%)$ fall in the North West quadrant (more costly, less effective). Figure 1B shows the probability that the intervention is cost-effective for a range of cost thresholds. At the cost threshold of $£ 100$ for an improvement of one point in FHT-AUC, there is $97.5 \%$ probability that steroid injection is cost-effective.

\section{Cost-utility analysis}

The mean health gain, expressed as a difference in mean QALYs between intervention and control groups over the three month follow-up period, was 0.0038 years, which is 1.4 quality-adjusted life days. We estimated the corresponding incremental cost-effectiveness ratio as $£ 20,840$ per QALY, by dividing the difference in mean cost of $£ 78.68$ between intervention and control groups (viz $£ 280.37$ - $£ 201.69$ ) by the difference in mean QALY of 0.0038 between the two groups (viz $0.1511-0.1473$ ).

We performed 5000 bootstrapped replications to generate a cost-effectiveness plane and a cost-utility acceptability curve. Figure $1 \mathrm{C}$ shows the cost-effectiveness plane with 3307 points (66.14\%) in the North East quadrant, 1686 (33.72\%) in the North West quadrant, 2 $(0.04 \%)$ in the South West quadrant and $5(0.10 \%)$ in the South East quadrant. Figure 1D is the corresponding cost-utility acceptability curve. Because $33.72 \%$ of the 
Table 3 Foot health thermometer scores and EQ-5D-3L utility index at baseline, 1 and 3 months

\begin{tabular}{|c|c|c|c|c|c|}
\hline \multirow[t]{3}{*}{ Outcome measure $^{a}$} & \multicolumn{5}{|c|}{ (Control $n=55$, Steroid $n=54$ ) } \\
\hline & \multicolumn{2}{|l|}{ Mean (SD) } & \multicolumn{2}{|c|}{ Mean adjusted for the baseline (SE) } & \multirow{2}{*}{$\begin{array}{l}\text { Estimated difference }{ }^{\mathrm{b}}[95 \% \mathrm{Cl}] \\
\text { significance }\end{array}$} \\
\hline & Control & Steroid & Control & Steroid & \\
\hline \multicolumn{6}{|c|}{ EQ-5D-3L utility index } \\
\hline Baseline & $0.5831(0.2947)$ & $0.5346(0.3023)$ & Not applicable & Not applicable & Not applicable \\
\hline 1 month & $0.6213(0.2523)$ & $0.6129(0.2717)$ & $0.6178(0.0351)$ & $0.6164(0.0354)$ & $-0.0014(-0.0976,0.1004) p=0.98$ \\
\hline 3 months & $0.5944(0.2852)$ & $0.6226(0.2771)$ & $0.5930(0.0388)$ & $0.6241(0.0388)$ & $0.0311(-0.0780,0.1403) p=0.57$ \\
\hline QALY & $0.1510(0.0522)$ & $0.1506(0.0511)$ & $0.1473(0.0062)$ & $0.1511(0.0068)$ & $0.0038(-0.0146,0.0221) p=0.68$ \\
\hline \multicolumn{6}{|c|}{ Foot health thermometer scores } \\
\hline Baseline & $48.17(23.68)$ & $45.23(21.22)$ & Not applicable & Not applicable & Not applicable \\
\hline 1 month & $49.30(24.21)$ & $60.28(22.01)$ & $48.49(3.07)$ & $60.81(3.10)$ & $12.32(3.66,20.98) p=0.006$ \\
\hline 3 months & $53.06(26.77)$ & $64.26(22.05)$ & $52.77(3.36)$ & $64.69(3.32)$ & $11.92(2.54,21.29) p=0.013$ \\
\hline FHT-AUC & $12.469(5.061)$ & $14.738(4.038)$ & $12.276(0.504)$ & $14.748(0.556)$ & $2.472(0.986,3.958) p=0.001$ \\
\hline
\end{tabular}

Imputed where necessary.

${ }^{b}$ In the final column, positive differences represent a better outcome for participants in the steroid group, and results in bold type are significant at $5 \%$ level.

bootstrapped estimates fall in the North West quadrant, where the intervention is more costly and less effective: this curve never reaches $70 \%$; and estimation of an upper limit for a two-sided 95\% CI is not possible. Therefore, only the bootstrapped one-sided 95\% lower confidence limit of $£ 2,370$ appears on the cost-effectiveness acceptability curve (Figure 1D). In the NICE threshold range of $£ 20,000$ to $£ 30,000$ per QALY [10], the probability that steroid injection is cost-effective lies between $49.7 \%$ and $54.9 \%$.

\section{Sensitivity analyses}

Firstly, we replaced the EQ-5D by the FHT-enhanced EQ-5D, and bootstrapped another 5000 replications to produce a third pair of cost-effectiveness plane and costeffectiveness acceptability curve. Figure $1 \mathrm{E}$ shows 4553 points $(91.06 \%)$ in the North East quadrant, 431 (8.62\%) in the North West quadrant, $16(0.32 \%)$ in the South East quadrant, but none at all in the South West quadrant. Figure $1 \mathrm{~F}$ presents the corresponding cost-utility acceptability curve. Again, the curve never reaches 1 because more than 125 (2.5\%) of bootstrapped estimates fall in the North West quadrant, and the costeffectiveness acceptability curve shows only the lower confidence limit of $£ 1,380$ (Figure 1F). The probability that steroid injection is cost-effective now lies between $80.8 \%$ and $84.6 \%$ over the NICE threshold range of $£ 20,000$ to $£ 30,000$ per QALY.

Secondly, we repeated the bootstrap and recalculated the cost-effectiveness acceptability curve and incremental cost-effectiveness ratio point estimate as if patients in the control group had attended for an outpatient appointment

Table 4 Mean frequency of health service use over three month follow-up period

\begin{tabular}{|c|c|c|}
\hline Count; mean, median, (min, max) & Control $(n=55)$ & Steroid $(n=54)$ \\
\hline \multicolumn{3}{|l|}{ NHS Secondary Sector: } \\
\hline \multicolumn{3}{|l|}{ Injection: } \\
\hline Lignocaine only & $55 ; 1.00,1.00(1,1)$ & $0 ; 0.00,0.00(0,0)$ \\
\hline Lignocaine \& steroid & $2 ; 0.04,0.00(0,1)$ & $54 ; 1.00,1.00(1,1)$ \\
\hline Outpatient consultation with ultrasound scan & $55 ; 1.00,1.00(1,1)$ & $54 ; 1.00,1.00(1,1)$ \\
\hline \multicolumn{3}{|l|}{ Outpatient follow-up visits: } \\
\hline Return visits & $4 ; 0.07,0.00(0,1)$ & $6 ; 0.11,0.00(0,1)$ \\
\hline \multicolumn{3}{|l|}{ Inpatient: } \\
\hline Inpatient hospital stay & $1 ; 0.02,0.00(0,1)$ & $23 ; 0.43,0.00(0,6)$ \\
\hline Accident and emergency & $4 ; 0.07,0.00(0,2)$ & $5 ; 0.09,0.00(0,2)$ \\
\hline \multicolumn{3}{|l|}{ NHS Primary Care Sector: } \\
\hline GP consultations & $54 ; 0.98,1.00(0,4)$ & $73 ; 1.35,1.00(0,12)$ \\
\hline Other health care practitioner consultations & $29 ; 0.53,0.00(0,9)$ & $35 ; 0.65,0.00(0,9)$ \\
\hline
\end{tabular}


Table 5 Mean cost of health service use $(£)$ over three month follow-up period

\begin{tabular}{|c|c|c|c|}
\hline NHS Secondary Sector: & $\begin{array}{l}\text { Control } n=55 \\
\text { mean (SD) in } £\end{array}$ & $\begin{array}{l}\text { Steroid } n=54 \\
\text { mean (SD) in } £\end{array}$ & $\begin{array}{l}\text { Mean difference in } £ \\
\text { (95\% Cl bootstrapped) }\end{array}$ \\
\hline \multicolumn{4}{|l|}{ Injection: } \\
\hline Lignocaine only & $0.25(0.00)$ & $0.00(0.00)$ & $-0.25(-)$ \\
\hline Lignocaine \& steroid & $0.14(0.71)$ & $3.74(0.00)$ & $3.60(-)$ \\
\hline Outpatient consultation with ultrasound scan & $145.74(0.00)$ & $145.74(0.00)$ & $0.00(-)$ \\
\hline \multicolumn{4}{|l|}{ Outpatient follow-up visits: } \\
\hline Return visits & $4.61(16.60)$ & $7.04(20.09)$ & $2.43(-4.52,9.43)$ \\
\hline \multicolumn{4}{|l|}{ Inpatient: } \\
\hline Inpatient hospital stay & $2.82(20.90)$ & $66.00(220.39)$ & $63.19(11.64,126.32) *$ \\
\hline Accident and emergency & $2.06(9.20)$ & $2.62(9.93)$ & $0.56(-3.05,4.22)$ \\
\hline Secondary care costs & $155.61(32.85)$ & $225.14(222.19)$ & $69.53(18.47,148.49) *$ \\
\hline \multicolumn{4}{|l|}{ NHS Primary Care Sector: } \\
\hline GP consultations & $31.57(35.77)$ & $39.95(56.52)$ & $8.37(-8.49,26.92)$ \\
\hline Other health care practitioner consultations & $14.51(43.06)$ & $15.28(40.55)$ & $0.77(-15.10,16.58)$ \\
\hline Primary care costs & $46.09(55.89)$ & $55.23(72.64)$ & $9.14(-14.68,33.91)$ \\
\hline Total costs & $201.69(67.74)$ & $280.37(246.74)$ & $78.67(18.25,152.34) *$ \\
\hline
\end{tabular}

NHS: National Health Service.

*Difference is significant at $5 \%$ level.

and received an injection, but no ultrasound scan. In this scenario the mean cost for the intervention group remained unchanged, while the mean cost for the control group fell from $£ 201.69$ (hospital outpatient visit with ultrasound-guided injection) to $£ 119.29$ (hospital outpatient visit with injection but no ultrasound guidance scan). Thus, the incremental cost-effectiveness ratio rose from $£ 31.83$ to $£ 65.16$ per point improvement on the FHT-AUC (difference in mean total costs, $£ 161.07$, divided by difference in mean FHT-AUC, 2.472), with a bootstrapped $95 \% \mathrm{CI}$ from $£ 33.47$ to $£ 172.49$. From the cost-effectiveness acceptability curve, the probability that steroid injection is cost-effective decreased to $85.2 \%$ at the cost threshold of $£ 100$ per point improvement in FHTAUC (Figure 2B), compared with $97.5 \%$ in the primary cost-effectiveness analysis (Figure 1B). Using QALYs, the incremental cost-effectiveness ratio point estimate rose from $£ 20,840$ to $£ 42,660$ per QALY; and the probability that steroid injection is cost-effective decreased to between $33.5 \%$ and $43.4 \%$ (Figure 2D) in the NICE threshold range of $£ 20,000$ to $£ 30,000$ per QALY, compared with 49.7\% and 54.9\% in Figure 1D.

Substituting the FHT-enhanced EQ-5D for the original EQ-5D-3L again brings the incremental costeffectiveness ratio below the lower UK threshold of $£ 20,000$, specifically to $£ 13,800$; and increases the probability of cost-effectiveness to $67.4 \%$ at the lower threshold of $£ 20,000$ and $77.2 \%$ at the higher threshold of $£ 30,000$ (Figure 2F).

\section{Discussion}

The MortISE trial with 131 participants found that foot health in the steroid group was significantly better than in the control group: the mean difference in the patientcentered Foot Health Thermometer (FHT) at 3 months was 14.1 scale points ( $95 \%$ CI: 5.5 to 22.8 points; $\mathrm{p}=$ 0.002) [8]. In the reduced sample of 109 participants for whom we had enough data for economic analysis, the mean difference fell to 11.9 scale points (95\% CI: 2.5 to 21.3 points; $\mathrm{p}=0.013$ ). When divided by the corresponding standard deviations of the baseline FHT scores, these differences yield 'effect sizes' of 0.62 and 0.53 respectively. Effect sizes greater than 0.5 are generally regarded as moderate and therefore worthwhile [30].

This economic evaluation supports and extends the findings from our clinical trial. If decision makers value an improvement of one point on the FHT over one year at $£ 100$, the upper confidence limit of the corresponding incremental cost-effectiveness ratio, then the use of ultrasound-guided steroid injection to alleviate foot pain from Morton's neuroma is cost-effective with a probability of $97.5 \%$; and a cost per point improvement on the FHT of only $£ 32$. In contrast, our secondary cost-utility analysis generated a cost per QALY of $£ 20,840$ and probability of cost-effectiveness little more than $50 \%$ across the recommended UK threshold, which ranges from $£ 20,000$ to $£ 30,000$ [10]. Though this estimated incremental costeffectiveness ratio is similar to that for using steroid treatment to manage sciatica [31], the discrepancy between 
(A) Foot Health Thermometer (FHT): CE plane

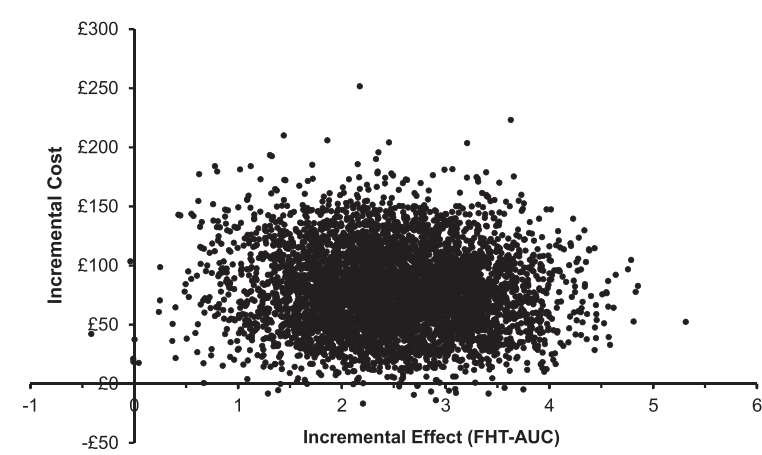

(C) QALY: CE plane

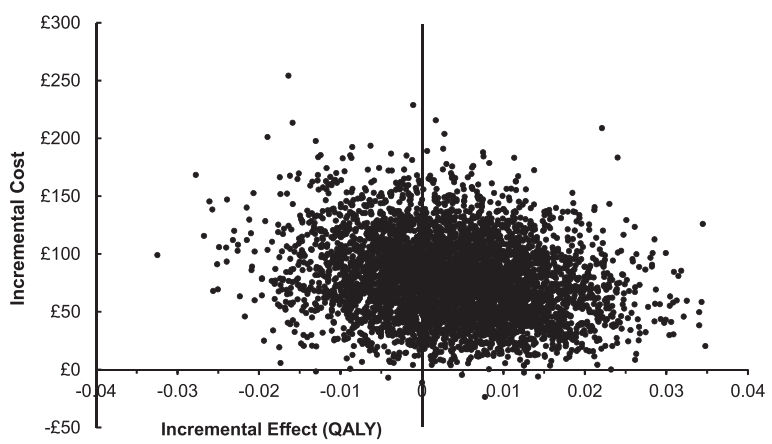

(E) FHT-enhanced QALY: CE plane

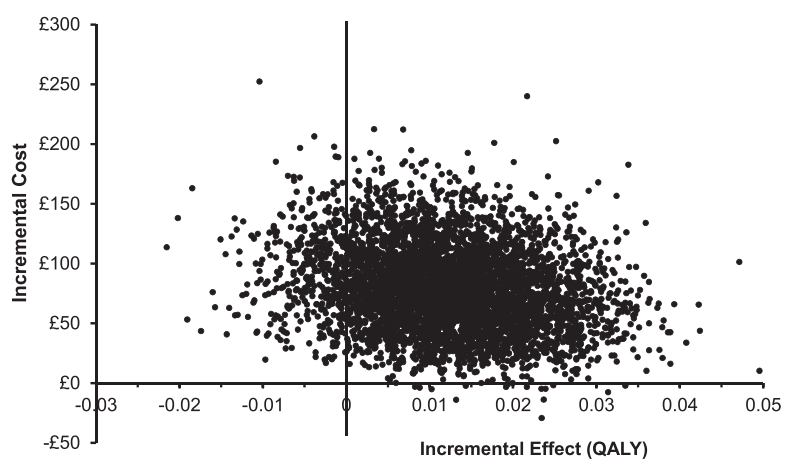

(B) Foot Health Thermometer (FHT): CEAC curve

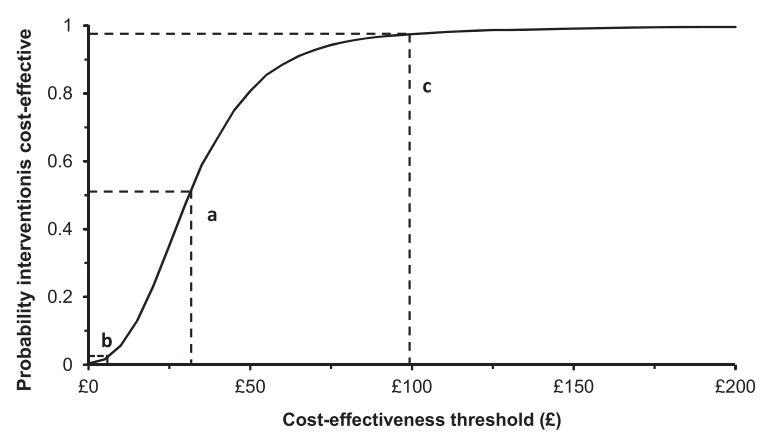

(D) QALY: CEAC curve

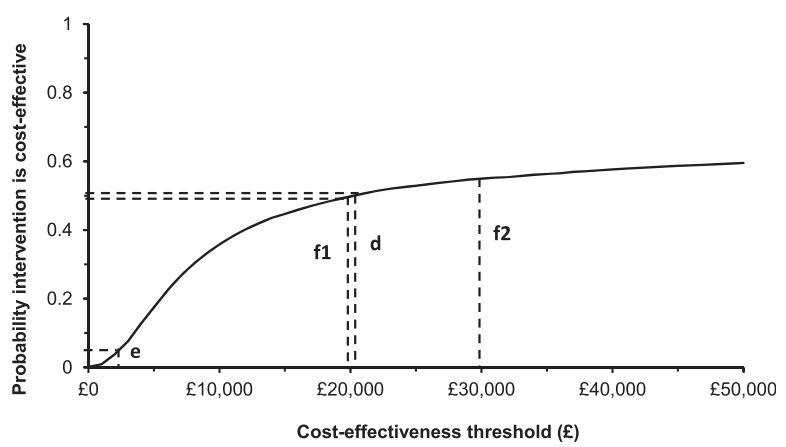

(F) FHT-enhanced QALY: CEAC curve

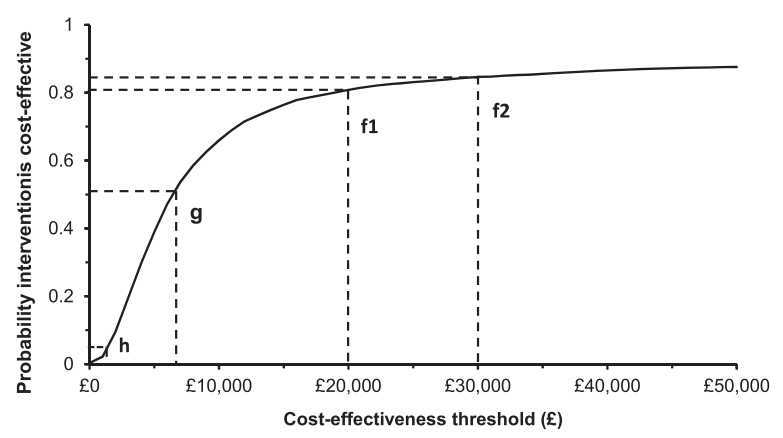

a - ICER point estimate ( $£ 32$ per one point improvement in FHT-AUC);

b - Lower limit of 95\% CI for Foot Health ICER (£7);

c - Upper limit of $95 \%$ CI for Foot Health ICER (£100);

d - ICER point estimate ( $£ 20,840$ per QALY);

e - One-sided lower $95 \%$ confidence limit $(£ 2,370)$;

f1 - Lower CE threshold $(£ 20,000)$;

f2 - Upper CE threshold $(£ 30,000)$

g - ICER point estimate ( $£ 6,400$ per QALY);

$\mathbf{h}$ - One-sided lower $95 \%$ confidence limit $(£ 1,380)$

Figure 1 Cost-effectiveness planes with 5,000 bootstrapped incremental cost-effectiveness ratio estimates for MortISE economic evaluation. Cost-effectiveness planes for Foot Health Thermometer (A), QALY (C) and FHT-enhanced QALY - 1st sensitivity analysis (E); and cost-effectiveness acceptability curves at three months for Foot Health Thermometer (B), QALY (D) and FHT-enhanced QALY - 1st sensitivity analysis (F). 
(A) Foot Health Thermometer (FHT): CE plane

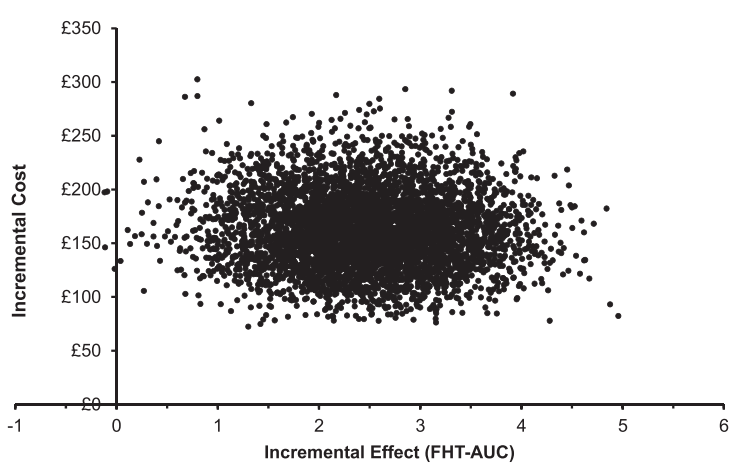

(C) QALY: CE plane

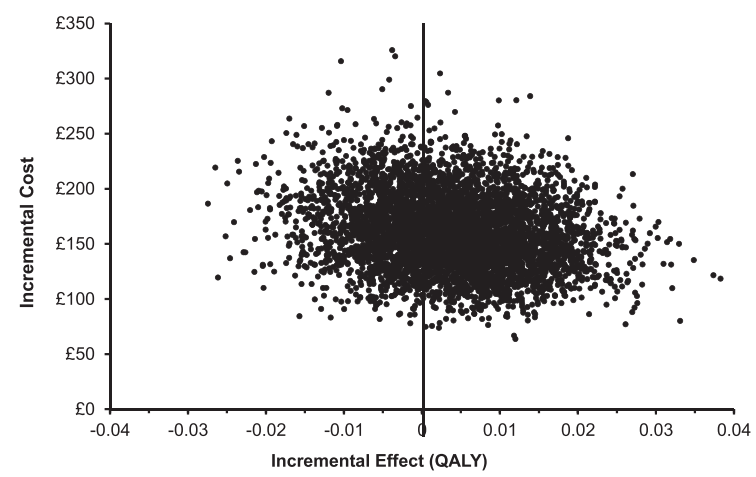

(E) FHT-enhanced QALY: CE plane

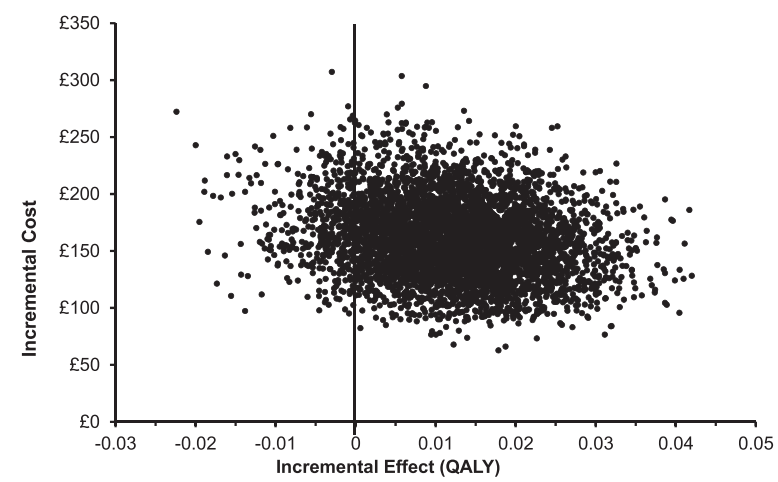

(B) Foot Health Thermometer (FHT): CEAC curve

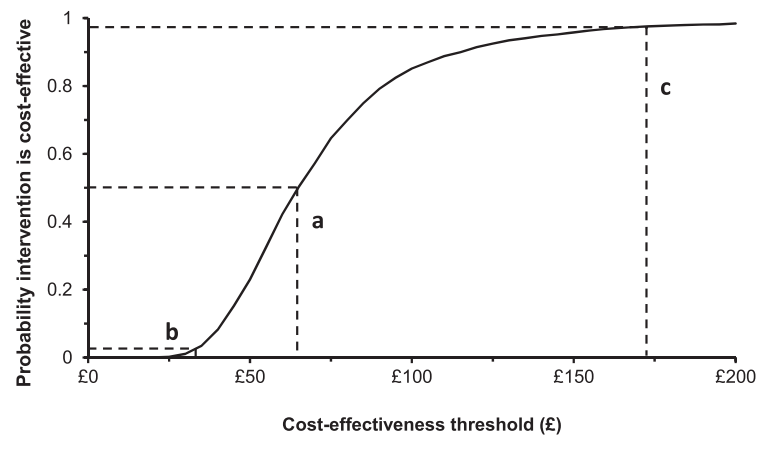

(D) QALY: CEAC curve

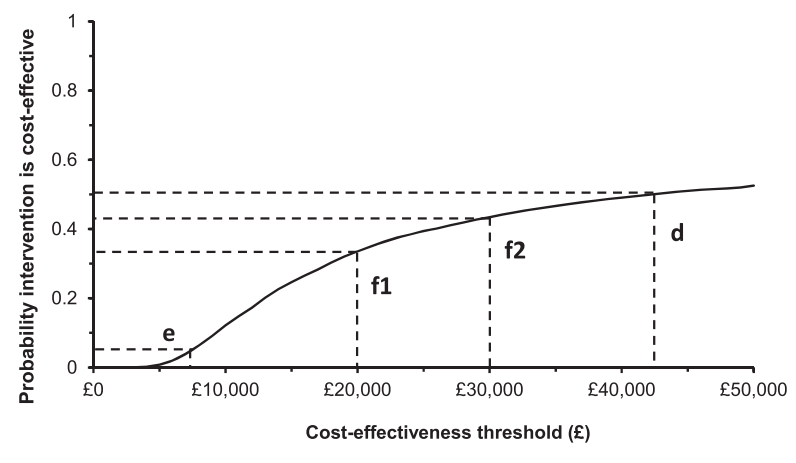

(F) FHT-enhanced QALY: CEAC curve

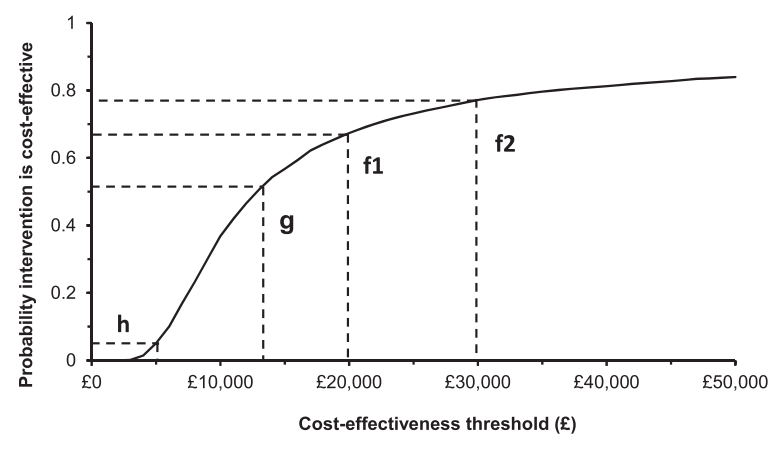

a - ICER point estimate ( $£ 65$ per improvement of one point in FHT-AUC);

b - Lower limit of $95 \%$ CI for Foot Health ICER (£33);

c - Upper limit of $95 \%$ CI for Foot Health ICER (£172);

d - ICER point estimate ( $£ 42,660$ per QALY);

e - One-sided lower $95 \%$ confidence limit $(£ 7,440)$;

f1 - Lower CE threshold $(£ 20,000)$;

f2 - Upper CE threshold $(£ 30,000)$

g - ICER point estimate ( $£ 13,800$ per QALY);

h - One-sided lower $95 \%$ confidence limit $(£ 4,980)$

Figure 2 Sensitivity analysis: cost-effectiveness planes with 5,000 bootstrapped incremental cost-effectiveness ratio estimates for MortISE economic evaluation. 2nd sensitivity analysis: cost-effectiveness planes for Foot Health Thermometer (A), QALY (C) and FHT-enhanced QALY (E); and cost-effectiveness acceptability curves at three months for Foot Health Thermometer (B), QALY (D) and FHT-enhanced QALY (F). 
our cost-effectiveness and cost-utility analyses alerted us to the danger that the latter had suffered from the known lack of responsiveness of the original EQ-5D [26,27].

We addressed this discrepancy by a sensitivity analysis that replaced the original EQ-5D by an enhanced version combining the economic validity of the EQ-5D with the greater responsiveness of the FHT. This reduced the incremental cost-effectiveness ratio to $£ 6,400$, well below the lower UK threshold of $£ 20,000$. This analysis also increased, to more than $80 \%$, the probability that steroid injections for Morton's neuroma are cost-effective. We believe this analysis, nominally secondary, has bridged the gap between our positive findings about effectiveness [8] and our initially negative findings about costeffectiveness. The strength of the association in our data between EQ-5D and FHT has convinced us that the strong evidence about effectiveness from the conditionspecific FHT translates into sufficient evidence about cost-effectiveness from the enhanced EQ-5D.

Our second sensitivity analysis indicates that, if control participants attend outpatient clinics and receive injection but no ultrasound scan, the incremental costeffectiveness ratio would rise from $£ 32$ to $£ 65$ per point improvement in FHT-AUC. This is still below our illustrative cost ceiling of $£ 100$. However, the cost per QALY would rise to $£ 42,660$, well above the higher UK threshold of $£ 30,000$.

As we are reporting findings after three months, we do not know whether the improvement in foot health was maintained, increased or reduced. As we explained in the main effectiveness paper of the MortISE trial [8], it is not appropriate to extrapolate effectiveness or cost-effectiveness findings beyond the period of data collection.

We believe this is the first cost-effectiveness study of steroid injections to alleviate pain from Morton's neuroma. As we cannot compare our findings with other cost-effectiveness analyses in Morton's neuroma, we have compared them with steroid treatment in managing sciatica.

The conclusions from this trial suffer from follow-up of only three months and our focus on direct costs to the National Health Service, rather than patient-borne costs. Though we would have preferred longer follow-up to assess the effect of steroid injection on subsequent surgical rates, the ethical committee insisted that we offer the intervention to participants in the control group after three months [8].

Though we asked participants about their own resource use, the self-reported client service receipt inventory is an accepted method of data collection when the recall period is only three months [32]. Furthermore, it facilitates the collection of data from many sources, as in this trial, where we are interested in both primary and secondary healthcare sectors. Hence, we see self- reporting in these patients as efficient use of research resources. For participants referred to private hospitals, we tried to obtain data on the resulting costs, but failed because those hospitals would not give us access to commercially sensitive data.

This trial has shown that steroid injection is effective and cost-effective for the National Health Service in treating Morton's neuroma in the short term. Any further trial of the management of Morton's neuroma should plan a longer follow-up period. It could also explore the effectiveness and cost-effectiveness of combinations of treatments, including the provision of insoles, analgesia and physiotherapy as well as steroid injection. It could also adopt a wider perspective to include patient-borne costs of attending hospital for treatment and of self-management of this condition.

\section{Conclusions}

Steroid injections are effective and cost-effective in relieving foot pain measured by the Foot Health Thermometer (FHT) for three months. However cost-utility analysis was initially inconclusive because the EQ-5D-3L is less responsive than the FHT to changes in foot health. By using the FHT to enhance the EQ-5D, we inferred that steroid injections yield good value in cost per QALY.

\section{Abbreviations}

$\mathrm{Cl}$ : Confidence interval; EQ-5D-3L: European quality of life-5 dimensions-3 levels; FHT: Foot health thermometer; FHT-AUC: Area under the curve of the foot health thermometer; FHT-enhanced EQ-5D: Foot health thermometerenhanced European Quality of life-5 Dimensions; NICE: National Institute for Health and Care Excellence; QALY: Quality-adjusted life year.

\section{Competing interests}

All authors have completed the Unified Competing Interest form at http:// www.icmje.org/coi_disclosure.pdf (available on request from the corresponding author) and declare: RTE and STY received grants from Bangor University via University of York (with transfer of Prof lan T Russell) in order to conduct the study; DR and ITR received grants from University of York in order to conduct the study; JNAG received grants from Scottish Government Chief Scientist's Office in order to conduct the study. CET, IB, DM and DJM declare that they have no competing interests.

\section{Authors' contributions}

RTE was the principal investigator responsible for economic analysis including the design, implementation, management of data collection and analysis. RTE also contributed to interpretation of results and drafted the first version of this manuscript. STY was the lead health economist responsible for the design, implementation, management of data (including entering and cleaning data), analysis and interpretation of results. STY has been actively involved in writing the manuscript and the critical revision of the paper. DR, the senior trial statistician, contributed to statistical analysis, interpretation of results and critical revision of the paper. CET, the primary investigator on the main clinical trial supported by the Chief Scientist Office (Scottish Government, Health and Social Care Directorate), managed and undertook the clinical trial and commented critically on the paper. IB, JNAG, DM and DJM managed and undertook the clinical trial, collected and coded data, and commented critically on the paper. ITR was responsible for trial design and commented critically on the paper. The initial draft of the manuscript was written by RTE and STY, but all authors have been involved in revising it critically for important intellectual content. All authors read and approved the final manuscript. 


\section{Authors' information}

RTE, Professor of health economics, BSC, MA, DPhil; STY, Research fellow in health economics, MSc, BSc(Hons); DR, Senior trial statistician, MA, PhD, DipMathStat, CStat; CET, Senior lecturer, PhD, BSc(Hons); IB, Consultant musculoskeletal radiologist, FRCPE, FRCR; JNAG, Consultant orthopaedic and trauma surgeon, MD, FRCSEd, FRCS(Tr\&Orth); DM, Research assistant, BSC (Hons); DJM, Professor of rehabilitation, DPhil, MSc, BSc(Hons); ITR, Professor of clinical trials, MA, MSc, PhD, HonDSc, FSS, MCMI, HonFRCGP, FRCPE, FFPH, FRCPL.

\section{Acknowledgements}

We acknowledge the continued support by Public Health Wales for the Centre for Health Economics and Medicines Evaluation, Bangor University. We thank the participants for taking part in the trial. We would like to thank Dr. Takashi Kikuchi and Dr. Natalia Hounsome for early stage analysis of data. Thank you to Eugenia Priedane for electronic scanning of some of the data. We would also like to thank Alison Shaw for providing reader support to the first author, RTE.

\section{Funding}

As stated in the main effectiveness paper [6], this project was funded by the Chief Scientist Office (Scottish Government, Health and Social Care Directorate). Funding was used for research costs only.

\section{Author details}

${ }^{1}$ Bangor University, Centre for Health Economics and Medicines Evaluation (CHEME), School of Healthcare Sciences, College of Health and Behavioural Sciences (CoHaBS), Ardudwy Hall, Normal Site, Bangor LL57 2PZ, Gwynedd, UK. ${ }^{2}$ Swansea University, Singleton Park, Institute of Life Science 2, College of Medicine, Swansea SA2 8PP, UK. ${ }^{3}$ Health Sciences, Queen Margaret University, Queen Margaret University Drive, Edinburgh EH21 6UU, Scotland, UK. ${ }^{4}$ The Royal Infirmary of Edinburgh, 51 Little France Crescent, Department of Radiology, Old Dalkeith Road, Edinburgh EH16 4SA, Scotland, UK. ${ }^{5}$ Musculoskeletal Directorate, The Royal Infirmary of Edinburgh, 51 Little France Crescent, Old Dalkeith Road, Edinburgh EH16 4SA, Scotland, UK. ${ }^{6}$ Teesside University, Health and Social Care Institute, Middlesbrough TS1 3BA, UK.

Received: 2 July 2014 Accepted: 9 February 2015

\section{Published online: 25 February 2015}

\section{References}

1. Thomson C, Campbell R, Wood A, Rendall G, et al. Disorders of the Adult Foot. In: Lorimer, editor. Neale's Disorders of the Foot. 6th ed. Edinburgh: Churchill Livingstone; 2001. p. 111-79.

2. Thomson CE, Gibson JNA, Martin D. Interventions for the treatment of Morton's neuroma. Cochrane Database Syst Rev. 2004;3(3):1-14.

3. Peng H, Swierzewski SJ III. Morton's Neuroma. http://www. healthcommunities.com/mortons-neuroma/about-mortons-neuroma.shtml. Published December 31, 1999.

4. Davis F. Therapeutic massage provides pain relief to a client with Morton's neuroma: a case report. Int J Ther Massage Bodywork. 2012;5(2):12-9.

5. Kilmartin TE, Wallace WA. Effect of pronation and supination orthosis on Morton's neuroma and lower extremity function. Foot Ankle Int. 1994; 15(5):256-62.

6. Gaynor R, Hake D, Spinner SM, Tomczak RL. A comparative analysis of conservative versus surgical treatment of Morton's neuroma. J Am Podiatr Med Assoc. 1989:79(1):27-30.

7. Hirschberg GG. A simple cure for Morton's neuralgia. J Am Podiatr Med Assoc. 2000:90(2):100-1.

8. Thomson CE, Martin DJ, McMillan D, Edwards RT, Russell D, Yeo ST, et al. Methylprednisolone injections for the treatment of Morton neuroma. J Bone Joint Surg Am. 2013:95:790-8.

9. Thomson CE, Gibson JNA, Martin D. Interventions for the treatment of Morton's neuroma. Cochrane Database Syst Rev. 2014;(Issue 11):CD003118. in press.

10. NICE. NICE Guide to the Methods of Technology Appraisal. London: NICE; 2007.

11. Drummond M, McGuire A. Economic Evaluation in Health Care. Oxford: Oxford University Press; 2001

12. Glick HA, Doshi JA, Sonnad SS, Polsky D. Economic Evaluation in Clinical Trials. Oxford: Oxford University Press; 2007.
13. Beecham J, Knapp M. Costing Psychiatric Interventions. In: Thornicroft G, Brewin C, Wing J, editors. Measuring Mental Health Needs. 1st ed. Oxford: Oxford University Press; 1992. p. 163-83.

14. Department of Health (DOH). NHS Reference Cost 2004-05. [http:// webarchive.nationalarchives.gov.uk/+/www.dh.gov.uk/en/ publicationsandstatistics/publications/publicationspolicyandquidance/ dh_4133221].

15. Curtis L, Netten A. Unit costs of health and social care 2005. Personal Social Services Research Unit (PSSRU): University of Kent; 2005.

16. Curtis L. Unit Costs of Health and Social Care 2012. Personal Social Services Research Unit (PSSRU): University of Kent; 2012.

17. British National Formulary. BNF 52, September 2006. London: BMJ Group and Pharmaceutical Press; 2006

18. Drummond MF, Sculpher MJ, Torrance GW, O'Brien BJ, Stoddart GL. Methods for the Economic Evaluation of Health Care Programmes ( $3^{\text {rd }}$ Edition). Oxford: Oxford University Press; 2005.

19. Brazier J, Jones N, Kind P. Testing the validity of the EuroQol and comparing it with the SF-36 health survey questionnaire. Qual Life Res. 1993;2:169-80.

20. EuroQoL Group. EQ-5D. [http://www.euroqol.org/].

21. Briggs $\mathrm{AH}$, Gray A. Handling uncertainty when performing economic evaluation of healthcare interventions. Health Technol Assess. 1999:3(2):1-134.

22. Fenwick E, O'Brien B, Briggs A. Cost-effectiveness acceptability curves - facts, fallacies and frequently asked questions. Health Econ. 2004;13:405-15.

23. Fenwick E, Marshall DA, Levy AR, Nichol G. Using and interpreting costeffectiveness acceptability curves: an example using data from a trial of management strategies for atrial fibrillation. BMC Health Serv Res. 2006;6:52.

24. Brooks R. Euroqol: the current state of play. Health Policy. 1996;37:53-72.

25. Petrou S, Hockley C. An investigation into the empirical validity of the EQ$5 \mathrm{D}$ and SF-6D based on hypothetical preferences in a general population. Health Econ. 2005;14:1169-89.

26. Wailoo A, Davis S, Tosh J. The Incorporation of Health Benefits in Cost Utility Analysis Using the EQ-5D: A Report by the Decision Support Unit. Sheffield: School of Health and Related Research, University of Sheffield; 2010

27. Lendrem D, Mitchell S, McMeekin P, Bowman S, Price E, Pease CT, et al. Health-related utility values of patients with primary Sjögren's syndrome and its predictors. Ann Rheum Dis. 2014;73(7):1362-8.

28. Herdman M, Gudex C, Lloyd A, Janssen MF, Kind P, Parkin D, et al. Development and preliminary testing of the new five-level version of EQ-5D (EQ-5D-5 L). Qual Life Res. 2011:20:1727-36.

29. Schwartz D, Lellouch J. Explanatory and pragmatic attitudes in therapeutic trials. J Chron Dis. 1967;20:637-48.

30. Cohen J. Statistical Power Analysis for the Behavioural Sciences. 2nd ed. London: Psychology Press; 1988

31. Price C, Arden N, Coglan L, Rogers P. Cost-effectiveness and safety of epidural steroids in the management of sciatica. Health Technol Assess. 2005;9(33):1-58.

32. Ridyard $\mathrm{CH}$, Hughes DA. Methods for the collection of resource use data within clinical trials: a systematic review of trials funded by the UK health technology assessment programme. Value Health. 2010;13:867-72.

\section{Submit your next manuscript to BioMed Central and take full advantage of:}

- Convenient online submission

- Thorough peer review

- No space constraints or color figure charges

- Immediate publication on acceptance

- Inclusion in PubMed, CAS, Scopus and Google Scholar

- Research which is freely available for redistribution 